\title{
SÍNTESE E CARACTERIZAÇÃo DE UM NOVO COMPLEXO DE PLATINA (IV) A PARTIR DE SEU ANÁLOGO DE PLATINA (II) UTILIZANDO IODO MOLECULAR COMO AGENTE OXIDANTE: UMA ROTA SINTÉTICA INTERESSANTE PARA OBTENÇÃO DE NOVOS COMPLEXOS DE PLATINA
}

\author{
Wendell Guerra, Heveline Silva, Mauro Vieira de Almeida e Ana Paula Soares Fontes* \\ Departamento de Química, Instituto de Ciências Exatas, Universidade Federal de Juiz de Fora, Campus Martelos, 36036-900 \\ Juiz de Fora - MG, Brasil \\ Recebido em 17/11/05; aceito em 7/4/06; publicado na web em 11/8/06
}

\begin{abstract}
SYNTHESIS AND CHARACTERIZATION OF A NOVEL PLATINUM (IV) COMPLEX FROM ITS PLATINUM (II) ANALOGUE USING MOLECULAR IODINE AS AN OXIDIZING AGENT: AN INTERESTING SYNTHETIC ROUTE FOR THE PREPARATION OF NEW PLATINUM COMPLEXES. In an attempt to reduce toxicity and widen the spectrum of activity of cisplatin and its analogues, much attention has been focused on designing new platinum complexes. This work reports the synthesis and characterization of novel compounds of the platinum (II) and platinum (IV) containing 2-furoic hydrazide acid and iodide as ligands. Although the prepared compounds do not present the classical structure of biologically active platinum analogues, they could be potentially active or useful as precursors to prepare antitumor platinum complexes. The reported compounds were characterized by ${ }^{1} \mathrm{H} N M R,{ }^{13} \mathrm{C}$ NMR, ${ }^{195} \mathrm{Pt} \mathrm{NMR}$, IR and elemental analyses.
\end{abstract}

Keywords: platinum complexes; hydrazides; iodo ligands.

\section{INTRODUÇÃO}

Cisplatina, cis-diaminodicloroplatina (II), uma das mais usadas drogas antitumorais, é bastante eficiente no tratamento de diversos tipos de câncer, em especial o de ovário e testículo e, em alguns casos, o índice de cura chega a aproximadamente $90 \%{ }^{1-4}$. Contudo, o efeito tóxico associado a este composto e o limitado espectro de atividade levou ao desenvolvimento de uma série de análogos, dentre eles carboplatina, oxaloplatina e nedaplatina, que estão disponíveis comercialmente, sendo que a nedaplatina só é usada no Japão. Em relação à cisplatina, a grande vantagem da carboplatina, da oxaloplatina e da nedaplatina diz respeito à diminuição da nefrotoxicidade, permitindo o uso de doses maiores. Entretanto, quanto ao espectro de atividade, elas são um pouco menos eficientes que a cisplatina 5 .

Vários outros novos complexos de platina estão sendo desenvolvidos e novos tipos estruturais têm emergido dos laboratórios de pesquisa. A alteração do grupo abandonador tem revelado resultados interessantes, inclusive na redução da toxicidade ${ }^{6,7}$.

Complexos antitumorais clássicos de platina apresentam ligantes abandonadores, sendo haletos e carboxilatos os mais comuns, e ligantes não abandonadores, como por ex., aminas, que acompanham o complexo até o interior da célula onde o mesmo interage com o DNA ${ }^{1,2,4}$. Neste sentido, a utilização de diferentes ligantes pode modificar a atividade biológica dos complexos.

Além de novos tipos de ligantes, outra estratégia utilizada tem sido a mudança da carga do metal, ou seja, a oxidação da platina (II) a platina (IV). Os complexos de platina (IV) têm sido muito estudados, pois são, em geral, mais solúveis em água que os análogos de platina (II), dentre outras características que despertam o interesse em seu estudo ${ }^{8-11}$.

Por outro lado, as hidrazidas e seus complexos também apresentam atividade biológica, como por ex., atividade fungicida e antibacteriana $^{12}$. Particularmente, nitrofurfural hidrazidas têm mos-

*e-mail: ana.fontes@ufjf.edu.br trado atividade antitumoral em vários tipos de células malignas in vitro e in vivo. Complexos de platina (II) com hidrazidas derivadas do ácido benzóico mostraram um forte efeito inibitório em células de leucemia in vitro, o que não foi verificado para os ligantes livres ${ }^{12}$.

Nosso grupo de pesquisa está interessado na obtenção de novos complexos de platina variando-se a natureza dos ligantes e o estado de oxidação do metal. Recentemente, foram publicados trabalhos envolvendo a preparação de complexos de cobre (II) e platina (II) com ácido 2-furóico hidrazida e cloro como ligantes ${ }^{6,13}$. O presente trabalho trata da síntese e caracterização de outros complexos da série, obtidos posteriormente. Os compostos aqui apresentados são de platina (II) e de platina (IV) e contêm ácido 2-furóico hidrazida e iodo como ligantes. Estes compostos podem vir a ser biologicamente ativos ou ser importantes como precursores para preparar novos complexos de platina com potencial atividade antitumoral.

\section{RESULTADOS E DISCUSSÃO}

O ligante utilizado neste trabalho, ácido 2-furóico hidrazida, foi obtido comercialmente e, para posterior comparação com o complexo, foi feita a caracterização por espectroscopia na região do infravermelho, $\mathrm{RMN}$ de ${ }^{1} \mathrm{H}$ e $\mathrm{RMN}{ }^{13} \mathrm{C}$.

Inicialmente, preparou-se o complexo de platina (II) de geometria cis contendo ácido 2-furóico hidrazida e iodo como ligantes, com o objetivo de utilizá-lo na síntese do complexo de platina (IV), seguindo o método descrito por Kaufman e Lowan ${ }^{14}$. Este método consiste da síntese de complexos de platina (IV) contendo iodo como ligantes a partir de complexos de platina (II), também contendo iodo como ligantes, e utilizando como agente oxidante uma solução etanólica de iodo a $2 \%$. O fato de o precursor de platina (II) também conter iodo como ligante evita a formação de diferentes tipos de complexos, uma vez que se o complexo de partida contiver cloro como ligante, poderá ser parcial ou totalmente substituído pelo iodo ${ }^{14}$.

Para a síntese do complexo de platina (II) contendo iodo, partimos do precursor $\mathrm{K}_{2}\left[\mathrm{PtI}_{4}\right]$ que foi preparado a partir do $\mathrm{K}_{2}\left[\mathrm{PtCl}_{4}\right]$ adicionando a este iodeto de potássio, conforme mostrado na parte experimental. 
Após a formação de $\mathrm{K}_{2}\left[\mathrm{PtI}_{4}\right]$, o ligante apropriado foi adicionado in situ, gota a gota, para obtenção do complexo de platina (II).

A síntese do complexo de platina (IV) foi feita de acordo com a metodologia descrita acima. Esta metodologia foi adotada como uma alternativa, em vez do método mais comum que consiste em utilizar o peróxido de hidrogênio como agente oxidante. Neste caso obteríamos complexos de platina (IV) contendo hidroxilas como ligantes. Contudo, quando utilizamos o peróxido de hidrogênio para a síntese do complexo de platina (IV) a partir de seu análogo contendo cloro, não obtivemos sucesso, possivelmente porque o peróxido de hidrogênio tenha oxidado o ligante, destruindo assim o complexo.

As condições de reação, como o tempo e a temperatura, foram determinadas experimentalmente. Os complexos obtidos, Figura 1, são sólidos, sendo o complexo de platina (II) amarelo escuro e o complexo de platina (IV) bege escuro, e ambos são solúveis em dimetilssulfóxido (DMSO) e dimetilformamida (DMF). Comparando o complexo de platina (II) contendo iodo como ligante ao seu análogo contendo cloro como ligante ${ }^{6}$, o primeiro apresentou rendimento bem maior e a reação de síntese se processou em uma escala de tempo cerca de duas vezes menor. Outra vantagem dos complexos contendo iodo é que este ligante é um bom grupo abandonador, podendo ser substituído por outros tipos de ligantes com facilidade.

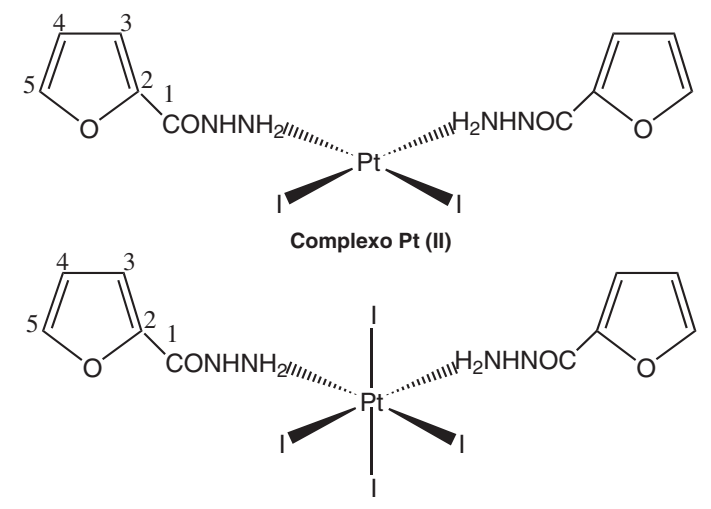

Complexo Pt (IV)

Figura 1. Complexos de platina (II) e platina (IV) sintetizados

Além da análise elementar e absorção atômica, os complexos foram caracterizados por espectroscopia na região do infravermelho, $\mathrm{RMN}$ de ${ }^{1} \mathrm{H}$ e RMN de ${ }^{13} \mathrm{C}$. Para o complexo de platina (II) também foi feita a caracterização por RMN de ${ }^{195} \mathrm{Pt}$.

Os resultados de absorção atômica para platina e análise elementar para carbono, hidrogênio e nitrogênio foram condizentes com as formulações propostas.

Os espectros de infravermelho para os complexos de Pt (II) e Pt (IV) são muito semelhantes e pode-se observar que a banda referente ao estiramento $\mathrm{NH}_{2}$ do ligante encontra-se melhor definida, deslocada e com menor intensidade quando comparado com o espectro do ligante livre. De $3260 \mathrm{~cm}^{-1}$ ela se desloca para 3224 e $3220 \mathrm{~cm}^{-1}$, respectivamente, para os complexos de $\mathrm{Pt}(\mathrm{II})$ e $\mathrm{Pt}(\mathrm{IV})$, indicando coordenação do metal neste grupo. Em $3080 \mathrm{~cm}^{-1}$ observa-se uma banda de absorção referente aos estiramentos dos grupos C-H do anel furânico. Em $1654 \mathrm{~cm}^{-1}$ no espectro do ligante aparece uma banda característica de estiramento de grupo $\mathrm{C}=\mathrm{O}$; no complexo de platina (IV), por ex., esta banda aparece em $1648 \mathrm{~cm}^{-1}$, um deslocamento de apenas $6 \mathrm{~cm}^{-1}$.

Bandas referentes ao estiramento $v_{\mathrm{Pt}-\mathrm{N}}$ são usualmente bandas fracas e difíceis de assinalar. Na literatura $\mathrm{v}_{\mathrm{Pt}-\mathrm{N}}$ tem sido localizado entre $530-700 \mathrm{~cm}^{-1}$. Os complexos aqui estudados apresentam novas bandas de absorção em $564 \mathrm{~cm}^{-1}$ para o complexo de $\mathrm{Pt}(\mathrm{II})$ e $586 \mathrm{~cm}^{-1}$ para o complexo de $\mathrm{Pt}(\mathrm{IV})$ que podem ser atribuídas a $\mathrm{v}_{\mathrm{Pt}-\mathrm{N}}{ }^{15}$. A banda referente ao estiramento C-O-C do anel furânico nos complexos encontra-se na mesma posição do ligante em $1017 \mathrm{~cm}^{-1}$.

Os espectros de RMN de ${ }^{1} \mathrm{H}$ e RMN de ${ }^{13} \mathrm{C}$ dos complexos de $\mathrm{Pt}$ (II) e Pt (IV) foram realizados. Para a caracterização do complexo de platina (IV), estes espectros não forneceram informações adicionais úteis, porém são coerentes com a estrutura proposta. Estes espectros foram feitos em dimetissulfóxido (DMSO) deuterado e, algumas vezes, observou-se o deslocamento parcial do ligante pelo solvente. Isto se deve à solvólise do complexo de platina em DMSO, sendo este fenômeno bastante comum e bem documentado na literatura $^{16-18}$.

No espectro de RMN de ${ }^{1} \mathrm{H}$ do complexo de $\mathrm{Pt}(\mathrm{II})$ observou-se um dupleto em $\delta 7,18$ corresponde ao hidrogênio $\mathrm{C} 3$ e um dupleto em $\delta 7,88$, referente ao hidrogênio $\mathrm{C} 5$. Um duplo dupleto em $\delta 6,65$ pode ser atribuído ao hidrogênio $\mathrm{C} 4$. Os hidrogênios do anel furânico não apresentaram deslocamento quando comparados ao ligante livre.

No espectro de RMN de ${ }^{13} \mathrm{C}$ não se observaram alterações significativas quando comparado ao do ligante livre. Os sinais em $\delta$ 111, 112, 144 e 147 são referentes aos carbonos C4, C3, C2 e C5, respectivamente. $\mathrm{O}$ carbono do grupo carbonila, $\mathrm{C} 1$, apresenta sinal em $\delta 152$.

$\mathrm{O}$ espectro de RMN de ${ }^{195} \mathrm{Pt}$ para o complexo de platina (II) mostra um sinal em $\delta$-3394. Este valor de deslocamento químico está condizente com complexos similares encontrados na literatura, nos quais a platina apresenta na sua esfera de coordenação dois átomos de iodo e dois átomos de nitrogênio ${ }^{19,20}$.

\section{PARTE EXPERIMENTAL}

Os reagentes e solventes foram usados sem purificação prévia.

Os espectros na região do infravermelho foram feitos no DQ da UFJF, em um espectrofotômetro Bomem FT IR MB-102, na região de 4000 a $300 \mathrm{~cm}^{-1}$, utilizando como suporte pastilhas de $\mathrm{KBr}$.

Os resultados de análise elementar de carbono, hidrogênio e nitrogênio dos compostos foram obtidos na Central Analítica do IQ da USP.

As análises por absorção atômica foram feitas em um espectrofotômetro modelo Hitachi 8200, no Departamento de Química da UFMG.

Os espectros de RMN de ${ }^{1} \mathrm{H},{ }^{13} \mathrm{C}$ e ${ }^{195} \mathrm{Pt}$ foram feitos no Laremar da UFMG utilizando um espectrômetro Brucker Avance DRX 200 e DRX 400. Os deslocamentos químicos foram expressos a partir do padrão interno TMS (RMN de ${ }^{1} \mathrm{H}$ ) e de uma solução de $\mathrm{K}_{2} \mathrm{PtCl}_{4}$ em $\mathrm{D}_{2} \mathrm{O}\left(\mathrm{RMN}{ }^{195} \mathrm{Pt}\right)$.

\section{Síntese dos complexos}

\section{Complexo de platina (II)}

À uma solução de $\mathrm{K}_{2} \mathrm{PtCl}_{4}(0,5 \mathrm{mmol} ; 0,2075 \mathrm{~g})$ em água (5 $\mathrm{mL})$ foi adicionado KI (2 mmol; $0,332 \mathrm{~g})$ dissolvido em água (5 $\mathrm{mL}$ ). A solução foi mantida sob agitação durante $30 \mathrm{~min}$. Após este tempo, foi adicionado in situ ácido 2-furóico hidrazida $(1 \mathrm{mmol}$; $0,126 \mathrm{~g}$ ) dissolvido em água $(5 \mathrm{~mL})$. A mistura reacional foi mantida sob agitação durante $16 \mathrm{~h}$. Após este tempo, filtrou-se o precipitado, que foi lavado com água e etanol, obtendo-se $0,340 \mathrm{~g}$ de produto, o que corresponde a um rendimento de $97 \%$.

IV $\vee \mathrm{KBr}\left(\mathrm{cm}^{-1}\right): 3224,3190,3139,3079,1646,1588,1568$, 1522, 1469, 1317, 1243, 1178, 1012, 885, 863, 754, 720, 590, 564, 529, 492, 483, 447, 416, 371, 332. Análise elementar calculada para $\mathrm{C}_{10} \mathrm{H}_{12} \mathrm{O}_{4} \mathrm{~N}_{4} \mathrm{PtI}_{2}$ : C 17,12; $\mathrm{H} 1,71 ; \mathrm{N} 7,99 ; \mathrm{Pt} 27,81$. Encontrada: C 17,10; H 1,65; N 7.99; Pt 26,91.

RMN de ${ }^{1} \mathrm{H}\left(200 \mathrm{MHz}\right.$; DMSO- $\left.d_{6}\right): \delta$ 7,18 e 7,88 (2d, hidrogê- 
nio $\mathrm{C} 3$ e hidrogênio $\mathrm{C} 5$, respectivamente); $\delta$ 6,65 (m, hidrogênio C4).

RMN de ${ }^{13} \mathrm{C}$ (50 MHZ; DMSO-d6): $\delta 111,112,144,147$ e 152 referentes aos carbonos $\mathrm{C} 4, \mathrm{C} 3, \mathrm{C} 2, \mathrm{C} 5$ e ao carbono do grupo carbonila, respectivamente.

RMN de ${ }^{195} \mathrm{Pt}\left(86 \mathrm{MHZ}\right.$; DMF- $\left.d_{7}\right): \delta-3394,06$.

\section{Complexo de platina (IV)}

Sob agitação e proteção da luz, foi feita uma suspensão do complexo de platina (II) $(0,350 \mathrm{~g}$; 0,5 mmol) em água (25 mL). Posteriormente, foram adicionados a esta suspensão $2 \mathrm{~mL}$ de solução de iodo a $2 \%$ em etanol. A mistura reacional foi mantida sob agitação durante $24 \mathrm{~h}$. Após este tempo, filtrou-se o precipitado, que foi lavado com água, metanol e etanol. Obteve-se 0,278 g de produto, correspondendo a um rendimento de $58 \%$.

IV $\vee \mathrm{KBr}\left(\mathrm{cm}^{-1}\right): 3480,3220,3190,3138,3079,1648,1588$, 1568, 1522, 1462, 1317, 1241, 1178, 1073, 1012, 947, 939, 885, 860, 754, 720, 595, 586, 536, 496, 448, 413, 350. Análise elementar calculada para $\mathrm{C}_{10} \mathrm{H}_{12} \mathrm{O}_{4} \mathrm{~N}_{4} \mathrm{PtI}_{4} \cdot \mathrm{H}_{2} \mathrm{O}: \mathrm{C} 12,33 ; \mathrm{H} 1,23 ; \mathrm{N} \mathrm{5,76}$; Pt 20,04. Encontrada: C 12,56; H 1,25; N 5.86; Pt 19,34.

\section{CONCLUSÕES}

Este trabalho descreveu a síntese de um novo complexo de platina (IV) a partir de seu análogo de platina (II) contendo iodo e ácido 2-furóico hidrazida como ligantes. A metodologia experimental utilizada constitui-se em uma ótima opção para obter complexos de platina (IV). Além disso, as reações para obtenção dos complexos contendo iodo como ligante foram feitas em uma escala de tempo menor e com um maior rendimento quando comparados à síntese dos análogos contendo cloro. Os complexos foram caracterizados pelas técnicas usuais de análise.

\section{AGRADECIMENTOS}

À FAPEMIG pelo apoio financeiro. W. Guerra e H. Silva agradecem ao CNPQ e à CAPES pelas bolsas concedidas.

\section{REFERÊNCIAS}

1. Zhang, C. X.; Lippard, S. J.; Curr. Opin. Chem. Biol. 2003, 7, 481.

2. Huq, F. J. Q.; Daghriri, H.; Beale, P.; J. Inorg. Biochem. 2004, 98, 1261.

3. Hidalgo, M.; Eckhardt, S. G.; J. Natl. Cancer Inst. 2001, 93, 178

4. Brabec, V.; Kasparkova, J.; Drug Resist. Update 2005, 8, 131.

5. Desoize, B.; Madoulet, C.; Crit. Rev. Onc. /Hemat. 2002, 48, 317.

6. Guerra, W.; Fontes, A. P. S.; de Almeida, M. V.; Silva, H.; Quim. Nova 2005, $28,809$.

7. Fontes, A. P. S.; de Almeida, S. G.; Nader, L.; Quim. Nova 1997, 20, 398.

8. Brandon, R. J.; Dabrowiak, J. C.; J. Med. Chem. 1984, 27, 861.

9. Kelland, L. R.; Drugs Future 1993, 18, 551.

10. Gund, A.; Keppler, B. K.; Angew. Chem., Int. Ed. 1994, 33, 186.

11. Matthew, D. W.; Trevor W. H.; Coord. Chem. Rev. 2002, 49, 67.

12. Dodoff, N.; Granharov, K.; Spassovska, N.; J. Inorg. Biochem. 1995, 60, 257.

13. Fontes, A. P. S.; Guerra, W.; Machado, F. C.; de Almeida, M. V.; Alves, W. A.; Ferreira, A. M. D. C.; Paduan-Filho, A.; Transition Met. Chem. 2004, 29, 382.

14. Kaufman, G. B.; Lowan, D. R.; Inorganic Syntheses 1973, 19, 234.

15. Nakamoto, K.; Infrared and Raman Spectra of Inorganic and Coordination Compounds, $3^{\text {rd }}$ ed., Wiley: New York, 1976.

16. Kerrison, S.; Sadler, P. J.; Inorg. Chim. Acta 1985, 104,197.

17. Fanizzi, F. P.; Intini, F. P.; Maresca, L.; Natile, G.; Inorg. Chem. 1990, 29, 29.

18. Farrel, N.; Kiley, D.; Schmidt, W.; Hacker, M.; Inorg. Chem. 1990, 29, 397.

19. Tessier, C.; Rochon, F. D.; Inorg. Chim. Acta 1999, 295, 25.

20. Rochon, F. D.; Buculei, V.; Inorg. Chim. Acta 2004, 357, 2218. 